\title{
Percutaneous endoscopic gastrostomy in head and neck cancer patients: indications, techniques, complications and results
}

\author{
Barbara F. Zuercher · Pierre Grosjean • \\ Philippe Monnier
}

Received: 12 August 2010/Accepted: 13 October 2010/Published online: 3 November 2010

(C) Springer-Verlag 2010

\begin{abstract}
The aim of this study was to review our experience in percutaneous endoscopic gastrostomy (PEG) performed in patients with cancer of the upper aerodigestive tract. Descriptive retrospective study of 142 patients (115 males, 27 females), mean age 62.4 years (25-84 years), with head and neck or esophageal cancer, who underwent PEG tube insertion between January 2006 and December 2008. The studied parameters were indications, success rate, rate and type of complications, and their management. Percutaneous endoscopic gastrostomy was inserted before chemoradiation therapy in $80 \%$ and during or after cancer treatment in $20 \%$ of the patients. PEG placement was possible in 137 patients (96\%). Major complications were observed in $9(7 \%)$ and minor complications in $22(17 \%)$ of the 137 patients. Seven of the 9 patients with a major complication needed revision surgery. The mortality directly related to the procedure was $0.7 \%$. Percutaneous endoscopic gastrostomy tube insertion has a high success rate. In patients with upper aerodigestive tract cancer, PEG should be the first choice for enteral nutrition when sufficient oral intake is not possible. Although apparently easy, the procedure may occasionally lead to severe complications. Therefore, a strict technique and knowledge of clinical signs of possible complications are mandatory.
\end{abstract}

Keywords Percutaneous endoscopic gastrostomy · PEG · Head and neck cancer

B. F. Zuercher $(\varangle) \cdot$ P. Grosjean · P. Monnier Department of Otorhinolaryngology, Head and Neck Surgery, University Hospital (CHUV),

1011 Lausanne, Switzerland

e-mail: barbarahauler@hotmail.com

\section{Introduction}

Introduced in 1980 by Gauderer et al. the procedure of percutanous endoscopic gastrostomy (PEG) has been established as the method of choice for enteral nutrition because of its rapidity and relative ease of insertion as well as its good tolerance and acceptance by the patients [1,2]. Different techniques of PEG placement have been reported, but the most widely used are either the pull method (Ponsky-technique), where the catheter is pulled through the upper digestive tract and out the abdominal wall $[1,3]$ or the push method (Sachs-Vine-technique), where a guidewire is placed into the stomach under endoscopic visualization and dilated with the Seldinger technique until the catheter is inserted through the abdomen into the stomach [3, 4]. In patients, suffering from a cancer of the upper aerodigestive tract, severe dysphagia leading to impaired or impossible oral intake occurs frequently before, during or after oncological treatment. In these situations, enteral feeding via a nasogastric tube, a PEG or radiological gastrostomy or even an open surgical gastrostomy becomes necessary. As pretherapeutic assessment of the tumor usually includes the examination of the upper digestive tract by endoscopy, PEG placement is usually the procedure choice in this population and is more and more taken over by ENT specialists in these patients.

However, serious and even lethal complications of this procedure are known to occur [5-7] and ENT specialists should be aware of the spectrum of these complications as well as of their early clinical manifestations. The aim of our study was to review our experience in PEG placement in patients with cancer of the upper aerodigestive tract, focusing on the analysis of the rate and type of complications in order to evaluate how they could possibly be prevented. 


\section{Materials and methods}

A retrospective study of 142 consecutive patients with a cancer of the upper aero-digestive tract who underwent PEG tube placement was conducted in the ENT Department of the University Hospital (CHUV) of Lausanne, Switzerland between January 2006 and December 2008. Approval from Institutional Review Board (IRB) was granted. The procedure was performed exclusively by ENT specialists.

The inclusion criteria were a history of upper aerodigestive tract cancer and a follow-up of at least 3 months after the procedure.

The collected demographic data included: age and sex of patient, site and stage of the tumor. The indication for PEG placement, the success or failure of PEG insertion, the number of puncture for successful placement, the use of prophylactic antibiotics, the number and type of complications and their management were also recorded. The relative risk of complications related to multiple punctures and to the lack of prophylactic antibiotics was calculated.

Complications were divided into major and minor ones. Major complication included either the need for revision surgery, the necessity of blood transfusion, or death of the patient within 30 days after the procedure. A complication was classified as minor if it could be managed by simple observation, local care at bedside or replacement of the gastrostomy tube.

\section{Technique of PEG insertion}

The standard pull method (Ponsky-technique) [3] was used in all patients. All procedures were performed by two surgeons, and under general anesthesia. Gastroscopy is performed with a standard videogastroscope. The stomach is inflated, so that its anterior wall will come in contact with the abdominal wall. The site of puncture is in the left epigastrium, at least $2 \mathrm{~cm}$ below the costal arch and usually about $2 \mathrm{~cm}$ paramedian to the left side. Its accurate location is determined by the point of maximal transillumination and maximal digital imprint on the anterior gastric wall as visualized by the endoscope. After the abdomen has been prepared and dressed, a first puncture with a fine needle (20G) is performed under continued aspiration until the needle tip is visualized in the stomach. The observation of air bubble in the syringe before seeing the needle tip in the stomach should alert on the possibility of bowel puncture. During withdrawal of the needle, local anesthetic is injected in the area around the puncture side. The pressure of the digital imprint is always maintained until the large-bore needle (trocar) is inserted into the gastric lumen under endoscopic control. Then, a pilot thread is placed through the trocar into the stomach and grasped by a biopsy forceps. In a single unit, the endoscope and the guidewire are withdrawn through the mouth. The end of the tube is fixed to the guidewire and pulled gently back down into the stomach and through the abdominal wall. If needed, a small cutaneous incision can be performed to ease the passage of the tube through the skin. The tube is fixed by an external bumper under adequate tension to avoid dislodgement of the gastric wall from the abdominal wall. A mark on the tube at the level of the skin is performed with a waterresistant pen to identify any postoperative dislodgement of the tube. The external end is covered by a sterile transparent dressing. A final control by endoscopy is performed to confirm the accurate position of the PEG-bumper and to rule out excessive bleeding or gastric perforation.

\section{Postoperative care}

Oral realimentation can be started $6 \mathrm{~h}$ after surgery with first liquid diet followed by normal diet after $13 \mathrm{~h}$. Enteral realimentation by the PEG includes $500 \mathrm{cc}$ of sterile saline solution between the 7 th and the 13 th hour postoperatively, followed by progressive enteral nutrition if well supported.

At day 6 postoperatively, the dressing is changed for the first time and the tube is mobilized vertically and by rotation to avoid impaction of the internal bumper into the gastric wall. Repositioning of the tube is made by traction on the tube until the mark performed during surgery appears at the skin level or according to the graduation on the tube. During the next 10 days, the sterile dressing is changed daily. After the 16th postoperative day, no dressing is necessary unless leakage is present, but local care with soap and daily mobilization of the PEG remains necessary.

\section{Results}

Our study included 142 patients. There were 115 males and 27 females with an average age of 62.4 years (25-82 years). None of these patients had preoperative contraindication to PEG placement such as coagulopathy, previous gastrectomy, ascites or morbid obesity.

\section{Indications for PEG placement}

In 113 of the 142 patients (79.6\%), PEG tube placement was indicated before primary (99 patients) or adjuvant (14 patients) chemoradiation therapy. In the remaining 29 patients (20.4\%), PEG was inserted because of long-lasting dysphagia, secondary to previous surgery (16 cases), extended tumor (11 cases) or to benign esophageal stenosis ( 2 cases). 
The majority of the patients (92\%) presented with an advanced stage of cancer (stage III or IV) in which $76 \%$ of the patients were localized in the oral cavity or the pharynx (Table 1). Eight patients underwent palliative oncologic treatment $(5.6 \%)$.

\section{Success}

Percutaneous endoscopic gastrostomy tube insertion was possible in 137 of the 142 patients (96\%). In five patients, the placement of PEG was not possible for the following reasons: lack of transillumination in three cases and impossibility to endoscopically reach the stomach because of a tight esophageal stenosis in two cases, respectively. In 126 patients, PEG was inserted after a single abdominal puncture, whereas 11 patients required two or more punctures for a successful insertion of the tube.

\section{Complications}

Nine patients $(7 \%)$ presented with a major complication (Table 2), requiring a surgical revision by laparoscopy or laparotomy in 7 of them. Despite surgical treatment, 1 of these 7 patients, suffering from a large iatrogenic gastric perforation supposed to be related to excessive inflation during PEG insertion, died. The mortality related to the procedure was, therefore, $0.7 \%$ (1/137). Another patient with preoperative gastric perforation was successfully treated by surgery. In the remaining 3 , surgical revision was indicated because of a pneumoperitoneum and peritonitis that occurred after secondary dislodgement of the PEG tube (Fig. 1). Postoperative course was uneventful in these three patients.

One patient, with immediate uneventful postoperative course presented a fecal discharge around the PEG tube 2 weeks after PEG insertion. A CT-scan of the abdomen showing interposition of the transverse colon between the abdominal wall and the stomach was diagnostic for a

Table 1 Tumor location and TNM stage

\begin{tabular}{llrrr}
\hline Location & \multicolumn{3}{l}{ Stage } & \\
\cline { 2 - 5 } & I & II & III & IV \\
\hline Oral cavity & 0 & 3 & 15 & 20 \\
Oro-hypopharynx & 0 & 8 & 27 & 46 \\
Larynx & 1 & 2 & 1 & 5 \\
Rhinopharynx & 0 & 0 & 0 & 5 \\
Esophagus & 0 & 0 & 5 & 1 \\
Unknown primary & 0 & 0 & 1 & 2 \\
Total & 1 & 13 & 49 & 79 \\
\hline
\end{tabular}

Table 2 Complications related to PEG placement $(N=137$ patients $)$

\begin{tabular}{ll}
\hline Major complications & $N=9(7 \%)$ \\
$\begin{array}{l}\text { Secondary displacement of the tube with } \\
\text { pneumoperitoneum or peritonitis }\end{array}$ & $3(2.2 \%)$ \\
Gastric perforation & $2(1.5 \%)$ \\
Esophageal perforation with mediastinitis & $1(0.7 \%)$ \\
Transcolic puncture & $1(0.7 \%)$ \\
Abscess/necrosis of the abdominal wall & $1(0.7 \%)$ \\
Abdominal wall bleeding & $1(0.7 \%)$ \\
Minor complications & $N=22(17 \%)$ \\
Severe abdominal pain & $9(6.6 \%)$ \\
Local infection & $8(5.8 \%)$ \\
Minor bleeding & $3(2.2 \%)$ \\
Secondary dislocation of the tube & $2(1.5 \%)$ \\
treated conservatively & \\
\hline
\end{tabular}

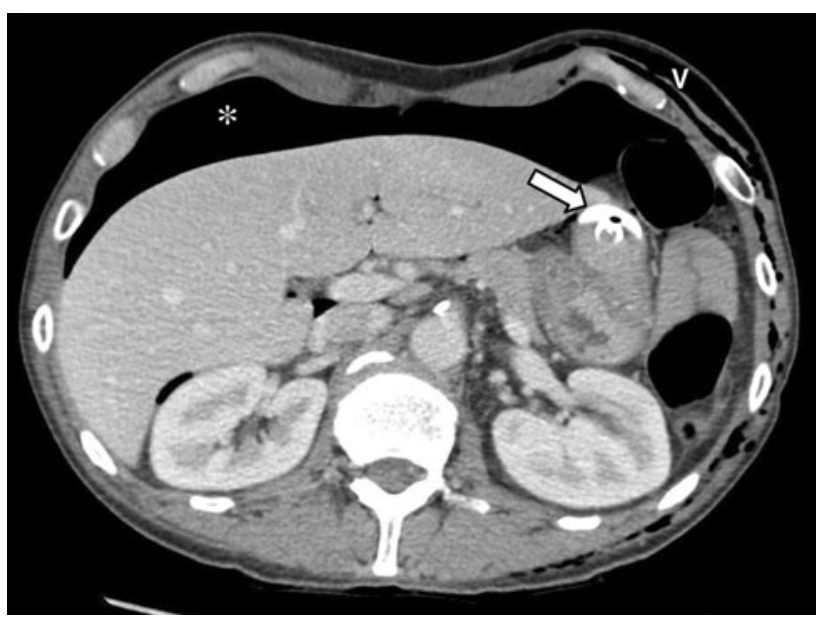

Fig. 1 Dislodgement of the PEG tube (arrow) with pneumoperitoneum (*) and subcutaneous emphysema (v)

gastrocolocutaneous fistula (Fig. 2). Surgical treatment with removal of the PEG and segmental colon resection led to complete recovery.

One patient presented with mediastinitis and pleural empyema after esophageal perforation following dilatation of a tumoral obstruction at the same time as PEG insertion. The patient was ineligible for major surgery but recovery was observed after prolonged intravenous antibiotic treatment.

Two patients with uncomplicated PEG insertion presented a major complication during the course of chemotherapy. One patient presented with severe abdominal wall bleeding secondary to chemotherapy-induced thrombocytopenia. Another patient with post-chemotherapy agranulocytosis developed abdominal wall necrosis and abscess that required surgical management. 


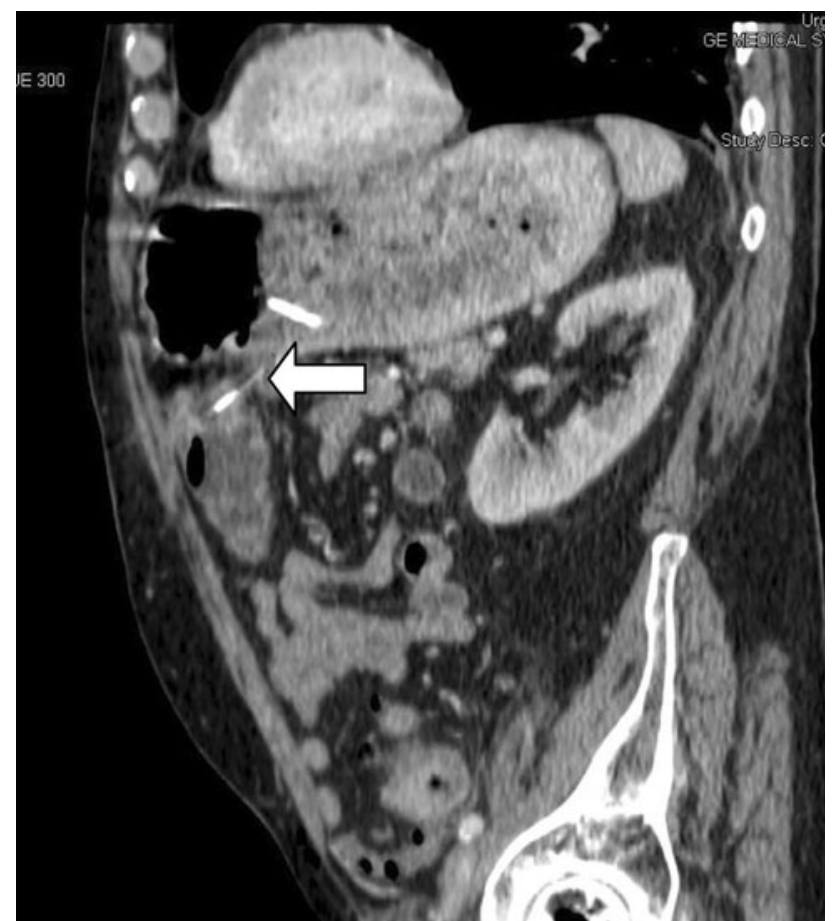

Fig. 2 Gastrocolocutaneous fistula with transcolic passage of the PEG tube (arrow)

Twenty-two patients (17\%) presented with a minor complications (Table 2). Nine patients complained of sudden onset of severe abdominal pain after mobilization, meal or the first dressing. The clinical evolution was favorable and enteral or oral feeding could be resumed after 1-2 days of observation. In two patients, who had similar symptoms, secondary dislocation of the tube was discovered by conventional radiography or CT-Scan. The treatment consisted of immediate repositioning of the tube with rapid clinical resolution of symptoms. We noted eight patients with a local infection, which could be treated with dressings or oral antibiotics.

\section{Prophylactic antibiotics}

Prophylactic antibiotics, mainly amoxicillin and clavulanic acid $(94.7 \%)$, followed by clarithromycin $(2.1 \%)$ and cefuroxime $(2.1 \%)$, were given in 95 patients. No prophylactic antibiotics were given in 41 patients. We could not get any information about intraoperative antibiotic prophylaxis for one patient.

Comparing the rate of complication in patients that received prophylactic antibiotics with those who did not, the relative risk of suffering a complication without antibiotics was 1.9 and 2.8 for major and minor complication, respectively.

Similarly, the relative risk of suffering from a major complication, when more than one puncture was required for PEG insertion, was 3.3.

\section{Discussion}

Percutaneous endoscopic gastrostomy is a well-established method for enteral feeding that may be indicated in a wide range of swallowing disorders: neurological disease, tumoral or long-lasting postoperative dysphagia [7]. In oncology, enteral alimentation by PEG is often used for patients with cancer of the upper aero-digestive tract, either during chemoradiation therapy or for prolonged posttreatment dysphagia.

In examining the predictive factors for the need of prolonged enteral feeding in patients with upper aerodigestive tract cancer, Gardine et al. [8] found that advanced cancer stage (mainly stage IV), primary pharyngeal tumors, combined treatment of surgery and radiotherapy and preoperative weight loss of more than $10 \mathrm{lb}$ may serve as indications for PEG insertion. In our group of head and neck cancer patients, the main indications for PEG placement were adjuvant or exclusive chemo-irradiation (79.6\%), oral or pharyngeal tumor location $(76 \%)$, and advanced disease (stage III or IV) (92\%). We proposed PEG placement as early as possible before the start of chemoradiation therapy and tried to avoid PEG insertion when the patient was at risk of developing altered blood counts secondary to chemotherapy. As a matter of fact, two patients in our series presented a major complication (bleeding and abdominal wall abscess and necrosis) related to chemotherapy-induced thrombopenia and agranulocytosis, respectively. Both of them had PEG placement just before the start of chemotherapy.

No patient in our series had PEG placement before tumor resection unless adjuvant chemoradiation was planned after surgery. Raynor et al. [9] showed an increased complication rate in patients who underwent PEG placement preoperatively and proposed the ideal time of PEG placement either during tumor resection or in the postoperative period, but not preoperatively. It should be, however, mentioned, that dysphagic patients with severe weight loss before diagnostic and management of head and neck cancer may benefit from preoperative enteral nutrition in order to decrease the rate of surgical complications [10]. In this group of patient and especially if prolonged oral feeding is likely to be impaired, PEG insertion seems to be the method of choice and can be inserted in the setting of pretherapy panendoscopy.

\section{Prophylactic antibiotics}

Different studies examining the benefit of antibiotic prophylaxis for PEG placement showed decreased incidence of wound infections when antibiotics were administered [11, 12]. Penicillin-based prophylaxis showed better results than cephalosporin prophylaxis $[11,12]$. In our series, 
prophylactic antibiotic therapy was given in 95 PEG placements. In accordance with the previous reports from the literature, we noticed an increased risk of 1.9 and 2.8 for major and minor complication, respectively, in patients without prophylactic antibiotics.

\section{Complications}

The technique of PEG insertion is relatively simple and the success rate in our series was $96 \%$. The reported success rate in the literature varies between 95 and $100 \%$ [13]. However, $7 \%$ of the patients in our series presented with major complications which correlates well with the rate reported in the literature $(1.1-17 \%)$ [5, 7, 13, 14]. Minor complication rates of $17 \%$ in our series also concurred with reported rates ranging from 8.3 to $65 \%[6,15,16]$. In our series, the procedure-related mortality was $0.7 \%$ and is in the range of those reported in the literature (1-2\%) [14].

One patient presented with a gastrocolocutaneous fistula due to transcolic puncture (Fig. 2). In the literature, the reported incidence of this severe complication may be as high as $2-3 \%$ [5]. Theoretically, the risk of this complication should be limited if

1. sufficient inflation of the stomach is performed, thus allowing close apposition of the stomach to the abdominal wall by pushing the large bowel and liver away.

2. the transillumination of the abdominal wall (Fig. 3) as well as the imprint of the finger (Fig. 4) are clearly visualized from outside and inside, respectively, and maintained until the trocar has been correctly inserted.

3. the first puncture is performed with a fine needle (20G) under continuous aspiration. If air is aspirated before endoscopic visualization of the needle in the stomach, a colic puncture must be suspected and the puncture

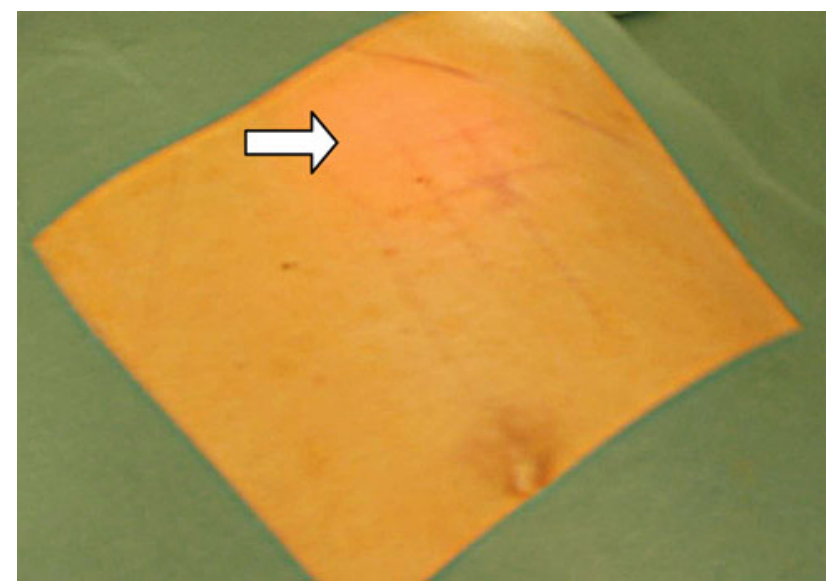

Fig. 3 Transillumination in the left epigastric region (arrow)

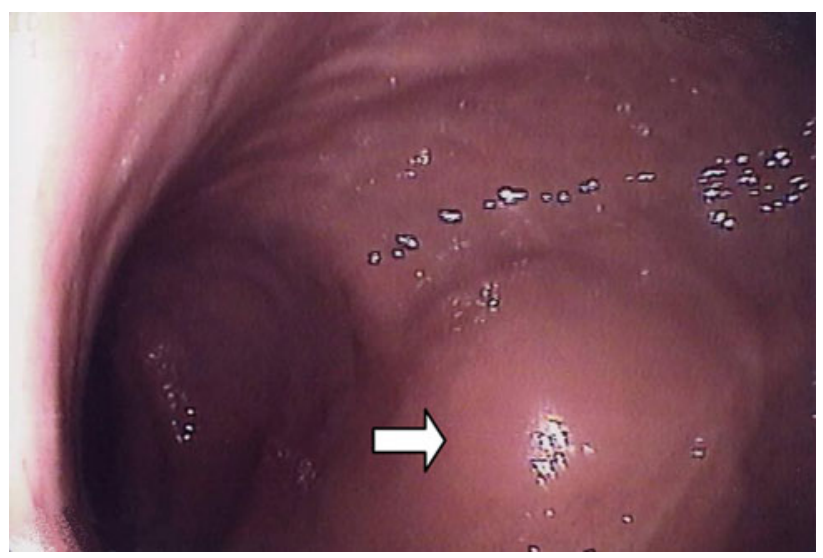

Fig. 4 Endoscopic view of the digital imprint (arrow)

has to be repeated in another direction before entering the trocar.

Whereas sufficient inflation of the stomach is likely to reduce the risk of transcolic puncture, it should be mentioned that overinflation may on the contrary increase this risk as described by Patwardhan [17]. In his series of 12 cases of gastrocolocutaneous fistula, the author noted that the puncture always occurred in the posterior wall of the stomach. This was also observed in our case (Fig. 2). It is hypothesized that excessive inflation of the stomach before PEG insertion, may cause an anterior rotation of the greater curvature including the gastrocolic omentum and the transverse colon which, therefore, lies between the stomach and the abdominal wall [18].

Friedmann et al. [19] reported 28 cases of PEG misplacement into the colon. Most patients (17/28) became symptomatic after blind tube replacement with sudden onset of severe watery diarrhea soon after feeding. Eleven of 28 patients presented fecal discharge in or around the tube between 3 and 75 days after PEG placement. Our patient also remained asymptomatic for 2 weeks before presenting with fecal discharge around the tube. It is important to keep in mind that this rare complication may become apparent only several weeks or months after PEG placement or when the tube is removed.

While overinflation may increase the risk of transcolic PEG insertion, it may also lead to gastric rupture as observed in one of our patient.

One patient in our series with synchronous head and neck and esophageal cancer presented with a perforation of the esophagus following dilatation of his tumoral stricture which remained undetected during the surgical procedure. He developed a mediastinitis which required prolonged antibiotic treatment alone as the patient was not eligible for major surgery. Therefore, in tumoral stricture of the esophagus, radiologic percutaneous gastrostomy, which generally does not require dilatation, should be considered 
the procedure of choice, unless the treatment is only palliative. In this case, PEG insertion can be combined with esophageal stent placement.

Inadvertent removal of PEG tube is a problem which occurs especially in disoriented patients, with an incidence of $1.6-4.4 \%$ [7, 20, 21]. We had no case of complete tube removal but recorded five cases of partial PEG dislodgement. In two patients, partial tube dislodgement was recognized early and immediate repositioning of the PEG tube by simple traction was followed by uncomplicated clinical course. In the other three patients, partial dislodgement of the PEG tube was only recognized after the patient developed acute abdominal pain with peritonitis or pneumoperitoneum. All three patients required surgical treatment. It should be noted that not all patients presenting acute abdominal pain after PEG insertion suffer from PEG dislodgement as illustrated by our group of nine patients with severe abdominal pain in whom no evidence of direct or indirect sign of PEG displacement could be demonstrated radiologically. Nevertheless, PEG displacement should always be excluded in such patients, especially if symptoms occur shortly after PEG mobilization or after the first enteral feeding. In this regard, marking the PEG tube at the level of the skin at the time of insertion when the correct position of the device can be assessed endoscopically provides an easy and reliable landmark of correct tube positioning in the postoperative period.

Although stomal tumoral seeding with development of abdominal wall metastases has been occasionally described in head and neck cancer patients undergoing PEG insertion by the pull technique, this seems to occur very rarely. Schrag [7] reported an incidence of less than $1 \%$ and Adelson [22] could only find 22 cases in a Medline search performed in 2004. Some authors recommend avoiding PEG insertion in patients with oropharyngeal or esophageal cancer [23]. Other authors proposed to use laparoscopy or open gastrostomy, or the push instead of the pull technique, to avoid tumor cell seeding [22]. No such complication was observed in our series and thus we presume that head and neck cancer should not be considered a contraindication to PEG insertion.

Finally, and although this was not the subject of our present study, it should be born in mind that enteral feeding with a PEG, although more invasive, presents many nutritional advantages compared to feeding with a nasogastric tube. In particular, several studies have demonstrated that in patients with head and neck cancer treated by radiation therapy, nutritional support via the PEG resulted in less weight loss, fewer hospitalizations for treatment-related complications, fewer interruptions of radiation, less treatment failures, and lower mortality [24] as compared to enteral feeding via nasogastric tube. In addition, the advantage of PEG feeding with regard to comfort and esthetic aspects are obvious $[25,26]$.

\section{Conclusion}

Percutaneous endoscopic gastrostomy placement has become the feeding modality of choice for patients with upper aerodigestive cancer who require mid- or long-term enteral nutrition. The surgical procedure is relatively simple but major complications may occur. A sound knowledge of these complications and especially of their early symptoms is mandatory. In addition, strict adherence to a rigorous surgical technique is essential to reduce the incidence of complications especially in a group of patients with frequent comorbidities.

Conflict of interest The authors declare that they have no conflict of interest.

\section{References}

1. Gauderer WL, Ponsky JL, Izant RJ (1980) Gastrostomy without laparotomy: a percutaneous endoscopic technique. J Pediatr Surg 15:872-875

2. Wiggenraad RGJ, Flierman L, Goossens A, Brand R, Verschuur HP, Croll GA, Moser LEC, Vriesendorp R (2007) Prophylactic gastrostomie placement and early tube feeding may limit loss of weight during chemoradiotherapy for advance head and neck cancer, a preliminary study. Clin Otolaryngol 32:384-390

3. DeLegge MH, Berry AJ (2008) Risks of endoscopic enteral access. Tech Gastrointest Endosc 10:36-44

4. Russell TR, Brotman M, Norris F (1984) Percutaneous gastrostomy: a new simplified and cost effective technique. Am J Surg 142:132-137

5. Khattak IU, Kimber C, Kiely EM, Spitz L (1998) Percutaneous endoscopic gastrostomy in paediatric practice: complications and outcome. J Pediatr Surg 33:67-72

6. Silas AM, Pearce LF, Lestina LS, Grove MR, Tosteson A, Manganiello WD et al (2005) Percutaneous radiologic gastrostomie versus percutaneous endoscopic gastrostomie: a comparison of indications, complications and outcome in 370 patients. Eur J Radiol 56(1):84-90

7. Schrag SP, Sharma R, Jaik NP, Seamon MJ, Lukaszczyk JL, Martin ND et al (2007) Complications related to percutaneous endoscopic gastrostomy (PEG) tubes. A comprehensive clinical review. J Gastrointestin Liver Dis 16:407-418

8. Gardine RL, Kokal WA, Beatty JD, Riihimaki DU, Wagman LD, Terz JJ (1988) Predicting the need for prolonged enteral supplementation in the patient with head and neck cancer. Am J Surg 156(1):63-65

9. Raynor EM, Williams MF, Martindale RG, Porubsky ES (1999) Timing of percutaneous endoscopic gastrostomy tube placement in head and neck cancer patients. Otolaryngol Head Neck Surg 120:479-482

10. Guo CB, Ma DQ, Zhang KH, Hu XH (2007) Relation between nutritional state and postoperative complications in patients with oral and maxillofacial malignancy. Br J Oral Maxillofac Surg 45(6):467-470 
11. Jafri NS, Mahid SS, Minor KS, Idstein SR, Hornung CA, Galndiuk S (2007) Meta-analysis: antibiotic prophylaxis to prevent peristomal infectin following percutaneous endoscopic gastrostomy. Aliment Pharmacol Ther 25:647-656

12. Gossner L, Keymling J, Hahn EG, Ell C (1999) Antibiotic prophylaxis in percutaneous endoscopic gastrostomy (PEG): a prospective randomized clinical trial. Endoscopy 31(2):119-124

13. Fang JC (2007) Minimizing endoscopic complications in enteral access. Gastrointest Endosc Clin N Am 17(1):179-196

14. Nicholson FB, Korman MG, Richardson M (2000) Percutaneous endoscopic gastrostomy: a review of indications, complications and outcome. J Gastroenterol Hepatol 15(1):21-25

15. Moller P, Lindberg CG, Zilling T (1999) Gastrostomy by various techniques: evaluation of indications, outcome, and complications. Scand J Gastroenterol 34(10):1050-1054

16. Preclik G, Grune S, Leser HG, Lebherz J, Heldwein W, Machka K, Holstege A, Kern WV (1999) Prospective, randomised, double blind trial of prophylaxis with single dose of co-amoxiclav before percutaneous endoscopic gastrostomy. BMJ 319(7214):881-884

17. Patwardhan N, McHugh K, Drake D, Spitz L (2004) Gastroenteric fistula complicating percutaneous endoscopic gastrostomy. J Pediatr Surg 39:561-564

18. Croaker GDH, Najmaldin AS (1997) Laparoscopically assisted percutaneous and endoscopic gastrostomy. Pediatr Surg Int $12: 130-131$

19. Friedmann R, Feldmann H, Sonnenblick M (2007) Misplacement of percutaneous inserted gastrstomy tube into the colon: report of 6 cases and review of the literature. JPEN J Parenter Enteral Nutr 31(6):469-476
20. Dwyer KM, Watts DD, Thurber JS, Benoit RS, Fakhry SM (2002) Percutaneous endoscopic gastrostomy: the preferred method of elective feeding tube placement in trauma patients. J Trauma 52:26-32

21. Larson DE, Burton DD, Schroeder KW, DiMagno EP (1987) Percutaneous endoscopic gastrostomy. Indications, success, complications and mortality in 314 consecutive patients. Gastroenterology 93:48-52

22. Adelson RT, Ducic Y (2005) Metastatic head and neck carcinoma to a percutaneous endoscopic gastrostomy site. Head Neck 27:339-343

23. Pickhardt PJ, Rohrmann CA Jr, Cossentino MJ (2002) Stomal metastases complicating percutaneous endoscopic gastrostomy: CT findings and the argument for radiologic tube placement. Am J Roentgenol 179:735-739

24. Lee JH, Machtay M, Unger LD et al (1998) Prophylactic gastrostomy tubes in patients undergoing intensive irradiation for cancer of the head and neck. Arch Otolaryngol Head Neck Surg $124: 871$

25. Hamidon BB, Abdullah SA, Zawawi MF, Sukumar N, Aminuddin A, Raymond AA (2006) A prospective comparison of percutnaeous endoscopic gastrostomy and nasogastric tube feeding in patients with acute dysphagic stroke. Med J Malaysia 61(1): 59-66

26. Norton B, Homer-Ward M, Donnelly MT, Long RG, Holmes GKT (1996) A randomised prospective comparison of percutaneous endoscopic gastrostomie and nasogastric tube feeding after acute dysphagic stroke. BMJ 312:13-16 